\title{
UM PROCEDIMENTO PARA ANÁLISE DE PERSISTÊNCIA NA VOLATILIDADE $\ddagger$
}

\author{
Marcelo Fernandes* \\ Marcos de Bustamante Monteiro**
}

\section{Resumo}

Diebold (1986) sugeriu que os modelos de variância condicional heterocedástica tendem a superestimar a persistência na volatilidade quando há mudanças na variância não-condicional. Um procedimento para analisar a persistência na volatilidade é aqui apresentado a partir de técnicas recentemente desenvolvidas na literatura de choques exógenos na variância. Estima-se inicialmente o melhor modelo de variância condicional heterocedástica possível e, em seguida, aplica-se aos resíduos padronizados o algoritmo ICSS (Inclán \& Tiao, 1994) buscando evidenciar a presença de instabilidades no segundo momento não-condicional. Incorporando variáveis dummies para representá-las, reestima-se o modelo para se obter uma estimativa mais confiável do grau de persistência de choques na volatilidade. $\mathrm{O}$ procedimento é aplicado com sucesso a algumaseséries financeiras brasileiras.

\section{Abstract}

Diebold (1986) suggested that conditional heteroskedastic models tend to overestimate persistence of volatility when there are instabilities at the unconditional second moment. We propose a procedure for analysing this phenomenon based on recent techniques developed by the sudden changes of variance literature. Applying the ICSS algorithm (Inclán \& Tiao, 1994) to the standardised residuals of the conditional heteroskedastic model, it is possible to detect break points at the unconditional

$\ddagger$ Os autores gostariam de agradecer a Osmani Guillen por gentilmente ter desenvolvido o prograrra para o algoritno ICSS. As sugestōes dos editores, emespecial, Joäo Victor Issler, e dois pareceristas anônimos foram de grande proveito, bem como œs comentários dos participantes do XVII Encontro Brasileiro de Econometria em Salvador, Bahia.

* École de Commerce Solvay e ECARE, Université Iibre de Bruxelles.

** Secretaria de Fazenda do Município do Rio de Janeiro.

\begin{tabular}{llllllll}
\hline R. de Econometria Rio de Janeiro & v. 17, $\mathrm{n}^{\mathrm{Q}}$ & 1, & pp. 15-43 & Maio 1997 \\
\hline
\end{tabular}


variance. By incorporating dummies to capture these instabilities, a more accurate and reliable estimate of the volatility persistence can be computed. The procedure is successfully applied to Brazilian financial data.

Palavras-Chave: Persistência na volatilidade, processos GARCH, algoritmo ICSS

Código JEL: C52, C22

\section{Introdução.}

Os modelos de variância condicional heterocedástica são reconhecidamente interessantes para analisar o comportamento da volatilidade nas séries financeiras, pois conseguem captar os fenômenos de agrupamento de volatilidade e leptocurtose característicos dessas séries. O modelo GARCH desenvolvido por Bollerslev (1986) obteve um grande sucesso na literatura, mas quase sempre indicando um elevado grau de persistência na volatilidade. Motivados pelas evidências empíricas, Engle \& Bollerslev (1986) desenvolveram o modelo IGARCH, onde a persistência na volatilidade é infinita, ou seja, a informação corrente permanece relevante para a previsão da variância condicional em qualquer horizonte futuro.

Apesar do sucesso do modelo IGARCH, Diebold (1986) e Lamoureux \& Lastrapes (1992) alertaram para o fato que a integração na variância pode ser apenas um reflexo de mudanças de regime não consideradas. Lastrapes (1989) investigou a influência de alterações da política monetária americana no processo estocásticogerador da taxa de câmbio de certas moedas em relação ao dólar americano. Os resultados mostraram que as trocas de regime implicavam a integração na volatilidade, de modo que a introdução de dummies para o intecepto da equação de segundo momento condicional melhorou o ajuste do modelo e reduziu o grau de persistência na variância. Na mesma linha, Lamoureux \& Lastrapes (1992) examinaram através de modelos GARCH a persistência de choques na volatilidade do retorno de ações negociadas no mercado americano. Associando variáveis 
binárias a 14 subamostras arbitrárias do conjunto de dados, reduziuse significativamente a meia-vida dos choques na volatilidade.

Outra alternativa para modelar o efeito GARCH com mudanças de regime consiste em utilizar o modelo SWARCH desenvolvido por Hamilton \& Susmel (1992), associando efeitos ARCH ao modelo de transição markoviana de Hamilton (1988). No modelo SWARCH, o efeito A RCH é estacionário em covariâncias em cada regime, com uma matriz de transição markoviana estabelecendo as probabilidades de mudança de regime. ${ }^{1}$ Deste modo, a variação do intercepto da equação do segundo momento condicional, que representa o choque na variância não-condicional, se torna endógena.

Este trabalho apresenta um novo procedimento para avaliar a persistência na volatilidade calcado na recente literatura de choques exógenos na variância. $\mathrm{O}$ procedimento exige uma estimação prévia de um modelo de variância condicional heterocedástica para filtrar a autocorrelação e heterocedasticidade condicional da série. Em seguida, aplica-se métodos estatísticos para detecção de possíveis pontos de troca de regime na variância e reestima-se o modelo inicial incluindo dummies para cada regime. Diferentemente da metodologia empregada por Lastrapes (1989) e Lamoureux \& Lastrapes (1992), onde as variáveis dummies são impostas arbitrariamente, o procedimento aqui exposto é isento, no sentido em que permite aos dados indicarem onde as variáveis binárias devem ser dispostas. Deste modo, a possibilidade de se introduzir variáveis binárias desnecessárias, que pode levar a uma subestimação da persistência na volatilidade, é mitigada. ${ }^{2}$

Em relação aos modelos SWARCH, esse método se mostra vantajoso no momento em que permite associar estruturas mais complexas

1 Por tornar a estrutura mito complexa, não se consegue associar os modelos de transição markoviana aos efeitos GARCH, de modo que se recomenda selecionar um número maior de defasagens para o modelo ARCH buscando captar toda a heterocedastidade condicional da série.

2 Emparticular, a introdução indiscriminada de variá veis dummies pode levar à estimasāo de uma persistência finita na volatilidade, mesmo quando o processo é realmente integrado. 
para a variância condicional. Em particular, modelos de volatilidade estocástica e processos mais complexos da família $\mathrm{ARCH}$ podem ser usados. Dentre os últimos, pode-se destacar os sofisticados APGARCH (Ding, Granger \& Engle, 1993), AGARCH (Hentschel, 1995) e EGARCH (Nelson, 1991), que especificam a variância condicional como função tanto da magnitude como do sinal dos erros passados.

Este trabalho segue a seguinte estrutura. Na seção 2, o procedimento da análise de persistência calcado em métodos de detecção de choques súbitos na variância é descrito. Na seção 3 , uma aplicação da metodologia desenvolvida para as principais séries financeiras brasileiras é apresentada, tentando captar os efeitos dos sucessivos planos econômicos lançados no Brasil. Finalmente, na seção 4, algumas conclusões e considerações a respeito dos resultados obtidos são tecidas.

\section{Análise de Persistência na Volatilidade.}

O modelo GARCH (Bollerslev, 1986) especifica uma dependência linear da variância condicional $h_{t}=E\left(e_{t}^{2} \mid L_{t-1}\right)$ em relação ao quadrado dos resíduos passados:

$$
h_{t}=\omega+\sum_{i=1}^{p} \alpha_{i} e_{t-i}^{2}+\sum_{j=1}^{q} \beta_{j} h_{t-j} .
$$

Para o modelo ser estacionário em covariâncias, a seguinte condição é suficiente $\sum_{i=1}^{p} \alpha_{i}+\sum_{j=1}^{q} \beta_{j}<1$. No caso de igualdade, tem-se o modelo IGARCH. Sem perda de generalidade, utiliza-se a seguir o modelo $\operatorname{GARCH}(1,1)$ para ilustrar o fenômeno de persistência na volatilidade. $O$ choque na volatilidade é definido como $\nu_{t} \equiv \varepsilon_{t}^{2}-h_{t}$ e denotando $\lambda \equiv \alpha+\beta$, chega-se à

$$
h_{t}=\omega+\alpha \nu_{t-1}+\lambda h_{t-1},
$$


que, após um processo de substituição recursiva, pode ser reescrita como:

$$
h_{t}=\sigma^{2}+\alpha\left(\nu_{t-1}+\lambda \nu_{t-2}+\lambda^{2} \nu_{t-3}+\cdots\right),
$$

onde $\sigma^{2}=\omega(1-\lambda)^{-1}$ é a variância não-condicional. A expressão (3) indica que a medida em que $\lambda \rightarrow 1$, o impacto dos choques passados na variância aumenta. No caso do modelo IGARCH, o segundo momento não-condicional é inexistente e a persistência dos choques é infinita.

Como assinalado por Diebold (1986), se houver uma instabilidade não considerada em $\omega$, a estimativa do parâmetro de persistência $\lambda$ tende a se aproximar da unidade. Deste modo, a realização de uma análise de persistência para a volatilidade é oportuna, objetivando encontrar possíveis choques na variância não-condicional. Inclán \& Tiao (1994) desenvolveram o algoritmo ICSS (Iterated Cummulative Sum of Squares) para deteç̧ão de pontos múltiplos de mudanças repentinas na variância. A aplicação do algoritmo nos resíduos padronizados do modelo GARCH estimado $a_{t} \equiv \hat{e}_{t} \hat{h}_{t}^{-1 / 2}$ permite identificar os períodos correspondentes a cada regime de variância não-condicional. Após associar variáveis binárias a cada regime (menos o primeiro, que serve de referência), estima-se o modelo novamente objetivando captar essas instabilidades. ${ }^{3}$

O método parte da soma cumulativa dos quadrados dos resíduos padronizados $C_{k}=\sum_{t=1}^{k} a_{t}^{2}$ para obter a soma cumulativa aproximadamente centrada dos quadrados:

$$
D_{k}=\frac{C_{k}}{C_{T}}-\frac{k}{T}
$$

onde $k=1,2, \cdots, T$ e $D_{0}=D_{T}=0$. Se a variância for homogênea, o valor de $D_{k}$ em relação à $k$ tende a ficar na vizinhança de zero.

3 O procedimento em duas etapas, que certarnente envolve urma perda de eficiência, não pode ser evitado, uma vez que, a priori, o número de quebras não é conhecido. 
Contudo, a presença de choques exógenos na variância, acarreta o distanciamento dos valores de $D_{k}$ em relação à zero. A partir da distribuição assintótica de $D_{k}$ sob a hipótese de homocedasticidade, ${ }^{4}$ pode-se construir um intervalo de confiança que, quando ultrapassado, indica a possibilidade de uma mudança de regime. Objetivando ganhos de eficiência, a procura pelos pontos de quebra é baseada no método iterativo abaixo.

Seja $a\left[t_{1}: t_{2}\right]$ a representação da série $a_{t_{1}}, a_{t_{1}+1}, \cdots, a_{t_{2}}\left(t_{1}<\right.$ $\left.t_{2}\right)$ e $D_{k}\left(a\left[t_{1}: t_{2}\right]\right)$ a soma cumulativa centrada dos quadrados no intervalo indicado. A partir da notação acima, o algoritmo pode ser expresso pelos seguintes passos:

(1) Considere $t_{1}=1$.

(2) Calcule $D_{k}\left(a\left[t_{1}: T\right]\right)$ e seja $k^{*}\left(a\left[t_{1}: T\right]\right)$ o argumento que maximiza:

$$
M\left[t_{1}: T\right]=\max _{t_{1} \leq k \leq T} \sqrt{\frac{T-t_{1}+1}{2}}\left|D_{k}\left(a\left[t_{1}: T\right]\right)\right| .
$$

Se $M\left[t_{1}: T\right]>D_{1-\rho}^{*}$, onde $\rho$ é o nível de confiança, considere $k^{*}\left(a\left[t_{1}: T\right]\right)$ um ponto possível de mudança da variância e passe ao próximo passo. Caso $\mathrm{M}\left[t_{1}: T\right]<D_{1-\rho}^{*}$, não há evidências de choques exógenos na variância e as iterações cessam.

(3) Considere agora $t_{2}=k^{*}\left(a\left[t_{1}: T\right]\right)$ e compute $M\left[t_{1}: t_{2}\right]$. Se $M\left[t_{1}: t_{2}\right]>D_{1-\rho}^{*}$, há um outro ponto provável de troca e este passo deve ser repetido até que $M\left[t_{1}: t_{2}\right]<D_{1-\rho}^{*}$, indicando que não existem mais evidências de quebras no intervalo $\left[t_{1}, t_{2}\right]$, de modo que o primeiro ponto possível de troca é $k^{\prime}=t_{2}$.

(4) Proceda do mesmo modo que no passo anterior para o restante da série, começando pelo ponto encontrado no primeiro passo

$4 \operatorname{Com} \sqrt{\frac{T}{2}} D_{k}$ se comporta assintoticamente como uma ponte brouniana, Inclán (1991) derivou $\propto$ valores assintóticos dos quantis de $\max \sqrt{\frac{T}{2}}\left|D_{k}\right|$ para a determinação dos intervalos de confiança. En particular, os valores críticos para os níveis de significância de $5 \%$ e $1 \%$ são respectivarnente $D_{.95}^{*}=1.358$ e $D_{.99}^{*}=1.628$. 
até a última observação. Então, sendo $t_{1}=k^{*}\left(a\left[t_{1}: T\right]\right)+1$, repita o procedimento até que $M\left[t_{1}: T\right]<D_{1-\rho}^{*}$. O último ponto provável encontrado é representado por $k^{\prime \prime}=t_{2}-1$.

(5) Se $k^{\prime}=k^{\prime \prime}$, há apenas um choque exógeno na variância e o algoritmo não precisa ser continuado. Caso contrário, repita os passos (2), (3), (4) e (5) para a parte central da série, isto é, $t_{1}=k^{\prime}$ e $t_{2}=k^{\prime \prime}$. Cada vez que os passos (2) e (3) forem repetidos, pode-se encontrar outros possíveis pontos de quebra. Denote por $N_{T}$ o número de pontos possíveis de choques na variância obtidos.

(6) Seja $c \nu=\left(c \nu_{0}, \cdots, c \nu_{N_{T}+1}\right)$ o vetor de todos os possíveis pontos de mudança na variância encontrados, onde $c \nu_{0}=0 \mathrm{e}$ $c \nu_{N_{T}+1}=T$. Cheque cada ponto possível de quebra $c \nu_{j}$, calculando $D_{k}\left(a\left[c \nu_{j-1}+1: c \nu_{j+1}\right]\right)$ para $j=1, \cdots, N_{T}$. Se $M\left[c \nu_{j-1}+1: c \nu_{j+1}\right]>D_{1-\rho}^{*}$, substitua o ponto testado pelo encontrado, caso contrário elimine-o. Repita o procedimento até que o número de pontos de choque na variância não se altere $e$ os pontos encontrados a cada nova iteração convirjam.

Normalmente, a convergência do algoritmo é bastante rápida, necessitando apenas de seis iterações aproximadamente. O critério de convergência pode ser estabelecido, por exemplo, como cada coordenada do vetor de pontos de quebra estar no máximo a duas observações da coordenada da iteração anterior. Na próxima seção, o algoritmo ICSS é aplicado para a análise de persistência da volatilidade de séries financeiras brasileiras, tentando captar instabilidades na variância não-condicional decorrentes dos planos de estabilização. 


\section{O Impacto dos Planos de Estabilização nas Volatilidades.}

Aplicações anteriores de modelos de variância condicional heterocedástica para séries financeiras brasileiras encontraram fortes evidências de um violento impacto dos planos de estabilização na volatilidade. ${ }^{5}$ Como um elevado grau de persistência na variância se mostrou sempre presente, é natural assumir que os planos de estabilização podem ter constituído choques repentinos na variância nãocondicional, levando à superestimação da persistência. Para avaliar se os planos econômicos são realmente o fator determinante para o excesso de persistência na volatilidade, aplicou-se o procedimento proposto na seção 2 para o logaritmo de algumas séries brasileiras: taxa de câmbio (dólar paralelo - fonte: Projeto Aries da FGV-RJ), taxa de inflação (medida a partir do retorno logaritmo do INPC fonte: Economática), taxa de juros (CDI over - fonte: CETIP) e índices de mercado (FGV-100 e IBOVESPA - fonte: Economática).

Excetuando a freqüência mensal da taxa de inflação, todas as demais séries são diárias. A série da taxa de câmbio consiste na cotação de venda do dólar no mercado paralelo do Rio de Janeiro entre 02/01/1989 a 10/03/1994, enquanto que a série do INPC se estende de janeiro de 1980 a fevereiro de 1995. A série da taxa de CDI over, IBOVESPA e FGV-100 correspondem aos respectivos intervalos de tempo: 11/03/1986 a 25/02/1994, 02/01/1986 a 28/10/1993 e 02/01/1989 a 10/03/1994.

5 Druck (1991) realizou um estudo de volatilidade para a taxa de inflaçāo; Fernandes (1994) investigou o cormportamento do indice da bolsa de valores do Rio de Janeiro; e Flôres, Monteiro \& Szafarz (1994) analisaram a taxa de câmbio nominal. 
Marcelo Fernandes \& Marcos de Bustamante Monteiro

Tabela 1. Resultados Preliminares ${ }^{6}$

Ordem de Integração, Normalidade e Homocedasticidade

\begin{tabular}{lccrc}
\hline \multicolumn{1}{c}{ Série } & PP & d & JB & $\mathbf{Q}^{2}(\mathbf{1 2})$ \\
\hline CDI & $-45,6699^{* *}$ & $-0,0925$ & $19.376,61^{* *}$ & $816,18^{* *}$ \\
dólar & $-40,9830^{* *}$ & 0,3605 & $8.391,76^{* *}$ & $618,63^{* *}$ \\
FGV-100 & $-34,4501^{* *}$ & 0,2223 & $3.772,82^{* *}$ & $429,91^{* *}$ \\
IBOVESPA & $-41,5261^{* *}$ & 0,0420 & $870,95^{* *}$ & $333,95^{* *}$ \\
INPC & $-3,1375^{*}$ & $0,4142^{*}$ & $196,42^{* *}$ & $263,64^{* *}$ \\
\hline \multicolumn{5}{c}{ * indica significância a $5 \%$} \\
\end{tabular}

Inicialmente, testes de ordem de integração, normalidade e heterocedasticidade são realizados para orientar a especificação dos modelos. O teste de Phillips \& Perron (1988) para raíz unitária e o método de Geweke \& Porter-Hudak (1983) - GPH - para estimação da ordem de integração foram usados por serem robustos a heterocedasticidade, em particular a efeitos $\mathrm{ARCH}$. O procedimento semiparamétrico de GPH foi adotado por considerar a possibilidade de ordem de integração fracionária, que está relacionada a propriedade de longa memória. ${ }^{7}$ A Tabela 1 apresenta os resultados de ambos os testes aplicados às primeiras diferenças de cada série, indicando que

$6 \quad P P$ consiste na estatistica do teste de Phillips-Perron para raiz unitária a partir da estatistica-t (incluindo constante) e truncando a matriz da variânciz-covariância de longo prazo na décirna-segunda defasagem Por sua vez, $d$ corresponde à ordem de integraçāo fracionária estimada pelo método de GPH, JB à estatística de Jarque-Bera para normalidade e $Q^{2}(12)$ ao teste de Ljung-Box para heterocedasticidade usando 12 defasagens.

7 Estudos de Monte Corlo mostram que os testes convencionais de raíz unitária não tem poder em relação a processes integrados fracionalmente, mesrmo quando esses são estacionários e inversiveis $\left(-\frac{1}{2}<d<\frac{1}{2}\right)$. 
a taxa de juros, ambos índices de mercado e o dólar são processos caracterizados por raíz unitária, mas a inflação parece ser integrada fracionalmente, embora estacionária. Após tomar as diferenças para tornar todas as séries $I(0)$, a homocedasticidade e normalidade de cada série foi analisada pelos testes de Ljung-Box e Jarque-Bera, respectivamente. Como reportado na Tabela 1 , as séries são heterocedásticas e não-normais, de modo que a aplicação de modelos de variância condicional heterocedástica é oportuna.

O procedimento utilizado para a seleção do modelo de variância condicional heterocedástica mais adequado para cada série é bastante simples. Estimou-se inicialmente por pseudo-máxima verossimilhança, ${ }^{8}$ o modelo $\operatorname{ARMA}(4,4)-\operatorname{GARCH}(2,2)$ :

$$
\begin{aligned}
& y_{t}=\varphi+\sum_{i=1}^{4} \phi_{i} y_{t-i}+\sum_{j=1}^{4} \theta_{j} e_{t-j}+e_{t} \\
& h_{t}=\omega+\sum_{i=1}^{2} \alpha_{i} e_{t-i}^{2}+\sum_{j=1}^{2} \beta_{j} h_{t-j} .
\end{aligned}
$$

Como processos $\operatorname{GARCH}(1,1)$ costumam ser adequados para séries financeiras, que em geral não possuem um alto índice de autocorrelação o modelo acima pode ser considerado suficientemente geral para uma especificação inicial. A defasagem menos significante foi sendo eliminada consecutivamente até todos os coeficientes serem estatisticamente diferentes de zero à um nível de $5 \%$ de significância. Em seguida, estimou-se o modelo selecionado acrescentando a res-

8 Bollerslev \& Wboldridge (1992) demonstraram a consistência e normalidade assintótica dos estimadores de pseudo-mávira verossimilhança para processos do tipo GARCH. Lurrsdaine (1996) mostrou que, ao contrário do caso de raíz unitária na média, a estimação dos parâmetros de um modelo IGARCH $(1,1)$ também são consistentes e assintoticamente normais. Portanto, a consideração de estatisticas-t para os parârnetros estimados é adequada mesmo quando a restrição de raíz unitária na variância é imposta. 
trição de raíz unitária na variância, ${ }^{9}$ objetivando comparar via o critério de informação bayesiano (BIC) os dois modelos (restrito e irrestrito). A especificação com o menor BIC foi então selecionada como a mais apropriada sem considerar choques exógenos na variância não-condicional.

Em seguida, o algoritmo ICSS foi computado para os resíduos padronizados de cada modelo estimado na busca de possíveis pontos de mudança de regime na variância. Introduzindo variáveis stepdummies de acordo com os resultados obtidos pelo algoritmo, os modelos foram reestimados e comparados com os modelos iniciais via BIC. Finalmente, em ambos os modelos, aplicou-se os testes de Ljung-Box para os resíduos padronizados e seus quadrados para verificar respectivamente a presença de autocorrelação e heterocedasticidade.

Em outras especificações, consideramos a presença do efeito calendário nas séries diárias de duas formas. A primeira via a normalização da variância condicional $h_{t}$ (e seus argumentos $e_{t-1}^{2}$ e $h_{t-1}$ ) pelo fator $n_{t}^{\kappa}$, onde $n_{t}$ é o número de dias sem negociação e $\kappa$ é um coeficiente a ser estimado (Noh, Engle \& Kane, 1994). O parâmetro $\kappa$ se mostrou significante em todas as séries exceto na taxa de câmbio (sempre por volta de 0,2 ). O segundo tratamento consistiu em acrescentar dummies para cada dia da semana tanto na média como na variância condicional. Aparentemente, as variâncias da taxa de juros e dos índices de mercado são mais elevadas nas segundas que nos demais dias da semana. O primeiro momento condicional independe do dia da semana em todas as séries diárias com exceção, talvez, do IBOVESPA, onde a variável binária é significativa a um nível de 10\%. Optamos por apresentar a seguir apenas os modelos mais sim-

9 Quando a variância condicional é descrita por um modelo GARCH(1, 1), como em todas as séries diárias aqui analicadas, a restrição que deve ser imp̣osta parase obter os processos integrados na variância $\operatorname{IGARCH}(1,1)$ equivale a $\beta=1-\alpha$. No caso da inflação, onde um ARCH(1) foi selecionado, a restrição implica a seguinte especificaşão para o segundo momento condicional $h_{t}=\omega+e_{t-1}^{2}$. 
ples (sem efeito calendário) por três motivos. Primeiro, os resultados qualitativos não se alteram se o efeito calendário é considerado ou não. Segundo, apresentando apenas os modelos mais parcimoniosos, a ilustração do procedimento proposto torna-se mais claro. Por último, Lumsdaine (1996) demonstrou a normalidade assintótica dos coeficientes estimados por pseudo-máxima verossimilhança apenas para urn modelo $\operatorname{IGARCH}(1,1)$ simples, sem qualquer variável exógena ou dummy na especificação da variância.

\section{- Taxa de Câmbio.}

O processo de seleção do modelo de variância condicional heterocedástica mais adequado para descrever o comportamento da volatilidade da taxa de câmbio resultou em uma especificação $\operatorname{ARIMA}(2,1,2)-\operatorname{IGARCH}(1,1)$. Deste modo, a persistência dos choques na variância é infinita, como pode ser visto pela meia-vida período necessário para que um choque tenha seu impacto reduzido pela metade.

O gráfico 1 da variância condicional deixa evidente o impacto dos planos econômicos implementados. Em especial, a volatilidade aumentou consideravelmente nos seguintes períodos: meados de 1989, janeiro de 1990, março de 1990 e janeiro de 1992, quando houve uma maxidesvalorização. A primeira data corresponde ao início do descongelamento de preços e à ameaça imediata de hiperinflação. A segunda refere-se aos efeitos das expectativas de enxugamento da liquidez um pouco antes do Plano Collor, que causou, por sua vez, o penúltimo pico de volatilidade.

$\mathrm{O}$ algoritmo ICSS determinou a presença de um choque exógeno na variância não-condicional no final de abril de 1992, fruto do otimismo político vivido a partir do impeachment do Presidente Fernando Collor de Melo, que reduziu substancialmente o nível de volatilidade $\left(\omega+\delta_{0} \sim 0,25 \omega\right)$. O novo modelo apresentou uma redução drástica da persistência na volatilidade, como ilustrado pela meiavida ao redor de 7 dias. Além dos testes de diagnóstico não evidencia- 
Marcelo Fernandes \& Marcos de Bustamante Monteiro

rem qualquer violação das hipóteses assumidas, o critério bayesiano confirmou a melhor especificação do modelo em relação ao previamente estimado (vide gráfico 2).

Tabela 2. Resultados para a Taxa de Câmbio ${ }^{10}$

\section{Coeficientes Antes ICSS Depois ICSS}

\begin{tabular}{lcc}
\hline$\varphi \times 10^{3}$ & $0,5641(3,6970)$ & $0,4332(2,9295)$ \\
$\phi_{1}$ & $0,3726(19,6883)$ & $0,7602(25,6856)$ \\
$\phi_{2}$ & $0,5809(23,3456)$ & $0,2049(6,8560)$ \\
$\phi_{3}$ & $-0,2727(-8,5757)$ & $-0,5968(-12,6565)$ \\
$\phi_{4}$ & $-0,5664(-15,7884)$ & $-0,2797(-5,9147)$ \\
$\omega \times 10^{3}$ & $0,0762(11,5761)$ & $0,0123(6,8764)$ \\
$\alpha$ & $0,3294(34,7361)$ & $0,4258(15,4941)$ \\
$\beta$ & $0,6706(70,7165)$ & $0,4592(38,2667)$ \\
$\delta_{0} \times 10^{3}$ & - & $-0,0090(-5,0499)$ \\
& $-\infty$, & 6,67 \\
\hline Meia-Vida & $-1.360,54$ & $-14.745,98$ \\
$L(\theta)$ & $-14.699,34$ & $21,920(0,1460)$ \\
BIC & $29,153(0,0230)$ & $12,993(0,5271)$ \\
$Q(16)$ & $7,1402(0,9292)$ & \\
$Q^{2}(16)$ & &
\end{tabular}

10 En cada tabela, a estatística-t consistente com heterocedasticidade é apresentada em parênteses logo após o parâmetro estimado, $\delta_{j}$ indica o coeficiente da dummay $j, L(\theta)$ indica o valor atingido pela função de verossinilhança e BIC assinala o critério de informaçāo bayesiano. Enseguida, os testes ljung-Box para os residuos padronizados e seus quadrados são apresentados com os correspondentes niveis atingidos de significância. 


\section{- Taxa de Inflação.}

Como o modelo ARFIMA $(1,0.4,0)$-IGARCH $(1,0)$ foi o mais adequado para a taxa de inflação, novamente a persistência na volatilidade se mostrou infinita. Apesar do modelo ter passado nos testes de diagnóstico propostos, o algoritmo ICSS encontrou evidências a favor de dois regimes na variância não-condicional. $O$ fracasso do Plano Cruzado no final de 1986 demarca o início de um período mais conturbado $\left(\omega+\delta_{0} \sim 9 \omega\right)$. A inspeção dos gráficos 3a e 4a mostram claramente a estabilidade da variação da inflação antes do Plano Cruzado. Tanto antes como após a aplicação do algoritmo ICSS, encontrou-se evidências de volatilidade exagerada na implementação dos Planos Collor e Real, como ilustrado nos gráficos 3a e 4a.

Tabela 3a. Resultados para a Taxa de Inflação

\section{Coeficientes Antes ICSS Depois ICSS}

\begin{tabular}{lcc}
\hline$\varphi$ & $0,0111(4,7337)$ & $0,0098(5,2926)$ \\
$\phi_{1}$ & $0,5902(27,0603)$ & $0,5800(24,0524)$ \\
$\omega \times 10^{3}$ & $0,5084(9,5137)$ & $0,1459(4,0880)$ \\
$\alpha$ & 1,0000 & 1,0000 \\
$\delta_{0} \times 10^{3}$ & $-\quad-$ & $0,9050(4,0430)$ \\
\hline Meia-Vida & $\infty$ & $\infty$ \\
$L(\theta)$ & 509,57 & 528,99 \\
BIC & $-1.003,61$ & $-1.037,27$ \\
$Q(16)$ & $13,462(0,6388)$ & $12,278(0,7246)$ \\
$Q^{2}(16)$ & $19,766(0,2310)$ & $7,2871(0,9673)$ \\
\end{tabular}


A introdução da variável binária trouxe apenas uma melhor especificação do modelo segundo o critério de informação bayesiano. A persistência na volatilidade permaneceu infinita, uma vez que o modelo onde a restrição $\alpha=1$ é válida continuou sendo a melhor representação. ${ }^{11}$

Uma explicação possivel para a excessiva persistência na volatilidade do processo inflacionário pode ser tecida nos moldes de Friedman (1977), isto é, o nível de volatilidade seria uma função também da magnitude da taxa de inflação. Como essa é caracterizada por longa memória, sua omissão poderia estar gerando viés na estimação dos parãmetros do segundo momento condicional. Para verificar essa hipótese, aplicamos a mesma metodologia de seleção do modelo iniciando com uma especificação um pouco mais geral, incluindo taxas de inflação passadas (até a quarta defasagem) na equação do segundo momento condicional. ${ }^{12}$ O modelo ARFIMA $(1,0,4,0)-\operatorname{IGARCH}(1,0)$ com uma defasagem da inflação se mostrou o mais apropriado em detrimento do modelo irrestrito explosivo $(\alpha=1,21)$. O algoritmo ICSS voltou a indicar um ponto de quebra em 1986, desta vez um pouco antes da implementação do Plano Cruzado. Apesar do modelo irrestrito não ter sido mais explosivo, a grande proximidade do caso integrado $(\alpha=0,99)$ determinou a preferência do critério bayesiano pelo modelo integrado na variância (ver Tabela $3 \mathrm{~b}$ ).

11 Os modelos irrestritos (sem a restrição $\alpha=1$ ), antes e depois do algoritno ICSS, são ambos explosivos (respectivamente $\alpha=1,48$ e $\alpha=1,32$ ), entretanto nota.se uma redução considerável do valor de $\alpha$ após a consideração da mudança de regines. Como o critério de informação bayesiano julgou os modelos restritos $(\alpha=1)$ mais apropriados que os irrestritos em ambos os casos, apresentarmas apenas os primeircs.

12 Issler (1991) apresentou evidências a favor da hipótese de Friedman ern relação ao processo inflacionário brasileiro usando urna especificação bastante similar para o segundo momento condicional, mas partindo de um nndelo rais estrutural para a dinânica da média condicional, onde se considera a relação de longo-prazo entre inflação e oferta monetária. Vale salientar que Issler (1991) considerou a inflação como um processo não-estacionário $I$ (1), enquanto que a inflação é vista aqui como um processo integrado fracionalmente de longa memória, porém estacionária. 
Um Procedimento para Análise de Persistência na Volatilidade

Tabela 3b. Resultados Alternativos para a Taxa de Inflação ${ }^{13}$

\section{Coeficientes Antes ICSS Depois ICSS}

\begin{tabular}{lcc}
\hline$\varphi$ & $0,0061(4,8621)$ & $0,0099(5,7386)$ \\
$\phi_{1}$ & $0,7090(40,0741)$ & $0,6121(24,0811)$ \\
$\omega \times 10^{3}$ & $-0,0352(-0,8938)$ & $-0,1574(-1,6712)$ \\
$\gamma$ & $0,0049(6,5839)$ & $0.0044(2,8448)$ \\
$\delta_{0} \times 10^{3}$ & $-\quad-$ & $0,3615(3,0570)$ \\
& & \\
\hline Meia-Vida & $\infty(\alpha=1)$ & $\infty(\alpha=1)$ \\
$L(\theta)$ & 530,32 & 534,42 \\
BIC & $-1.039,94$ & $-1.042,90$ \\
$Q(16)$ & $11,395(0,7845)$ & $10,478(0,8405)$ \\
$Q^{2}(16)$ & $8,1587(0,9440)$ & $7,2871(0,9673)$ \\
\hline
\end{tabular}

\section{- Taxa de Juros.}

O modelo $\operatorname{ARIMA}(2,1,0)-\operatorname{IGARCH}(1,1)$ estimado inicialmente, apresentou quatro pontos de quebra no segundo momento nãocondicional de acordo com o algoritmo ICSS. O Plano Collor demarcou o fim do primeiro regime, que se caracterizou por baixa volatilidade $\left(\delta_{0}<0\right)$, iniciando um período mais conturbados $\left(\delta_{1}>0\right)$. A maxidesvalorização do início de 1991 decretou o fim do segundo regime, enquanto que o impeachment trouxe o término do terceiro. A partir de dezembro de 1992, a volatilidade chegou ao seu patamar mais baixo $\left(\omega+\delta_{3} \sim 0,125 \omega\right)$.

13 O parâmetro $y$ mensura a influência da inflação passada na volatilidade corrente. 
Marcelo Fernandes \& Marcos de Bustamante Monteiro

Tabela 4. Resultados para a Taxa de Juros

\section{Coeficientes Antes ICSS Depois ICSS}

\begin{tabular}{|c|c|c|}
\hline$\varphi$ & $0,0007(4,5458)$ & $0,0062(2,6971)$ \\
\hline$\phi_{1}$ & $0,1527(6,4748)$ & $0,1749(6,6321)$ \\
\hline$\phi_{2}$ & $-0,0472(-2,0988)$ & $-0,0545(-2,2093)$ \\
\hline$\omega \times 10^{3}$ & $0,0071(18,3848)$ & $0,2660(7,3082)$ \\
\hline$\alpha$ & $0,2530(35,7308)$ & $0,2843(13,8862)$ \\
\hline$\beta$ & $0.7470(105,4977)$ & $0,6873(44,9668)$ \\
\hline$\delta_{0} \times 10^{3}$ & $-\quad-$ & $-0,1661(-5,0188)$ \\
\hline$\delta_{1} \times 10^{3}$ & - & $0,0692(1,8875)$ \\
\hline$\delta_{2} \times 10^{3}$ & - & $-0,1692(-4,6481)$ \\
\hline$\delta_{3} \times 10^{3}$ & - & $-0,2373(-6,7161)$ \\
\hline Meia-Vida & $\infty$ & 25,12 \\
\hline$L(\theta)$ & $7.360,54$ & $7.391,78$ \\
\hline $\mathrm{BIC}$ & $-14.704,64$ & $-14.750,68$ \\
\hline$Q(16)$ & $29,259(0,0220)$ & $22,955 \quad(0,1150)$ \\
\hline$Q^{2}(16)$ & $8,7353(0,8476)$ & $5,9101(0,9687)$ \\
\hline
\end{tabular}

Após a consideração dos choques, a persistência na volatilidade decresceu bastante, porém não tanto como os casos anteriores, uma vez que a meia-vida continuou razoavelmente elevada (cerca de um mês). Os picos de volatilidade para a taxa de juros foram similares aos das taxas de câmbio, podendo se destacar o impacto da ameaça de hiperinflação no final de 1989 e os Planos Cruzado II, Collor I e Collor II (vide gráficos 5 e 6).

\section{- Índices de Mercado.}

O modelo $\operatorname{ARIMA}(1,1,0)-\operatorname{GARCH}(1,1)$ sem intercepto na equação da média foi selecionado para descrever o comportamento 
do IBOVESPA. Apesar de $\lambda$ ser próximo da unidade, o critério de informação bayesiano selecionou o processo $\mathrm{GARCH}$ em detrimento ao modelo integrado na variância condicional. Como o algoritmo ICSS não encontrou evidências de qualquer choques exógeno na variância condicional, assegurou-se a confiabilidade da meia-vida de 10 dias estimada.

Tabela 5. Resultados para o IBOVESPA

\section{Coeficientes Antes/Depois ICSS}

\begin{tabular}{lc}
\hline$\varphi$ & $0,1360(5,4397)$ \\
$\omega \times 10^{3}$ & $0,1441(7,1143)$ \\
$\alpha$ & $0,1303(9,3102)$ \\
$\beta$ & $0,7932(44,9072)$ \\
\hline Meia-Vida & 9,71 \\
$L(\theta)$ & $5.182,52$ \\
$Q(16)$ & $16,736(0,4030)$ \\
$Q^{2}(16)$ & $5,8920(0,9692)$ \\
\hline
\end{tabular}

A correspondência entre os períodos de alta volatilidade e os planos econômicos foi mais uma vez uma constante, especialmente após o Cruzado, Verão, Collor e Collor II, como evidenciado no gráfico 7. A maxidesvalorização do final de 1987 também teve um forte impacto na volatilidade, bem como a ameaça de hiperinflação no segundo semestre de 1989. O comportamento da volatilidade do índice FGV-100 apresentou um maior grau de instabilidade que o IBOVESPA, uma vez que três pontos de quebra foram identificados: março de 1990, janeiro de 1991 e dezembro de 1992. Os picos de volatilidade mais expressivos refletem os efeitos de praticamente os mesmos eventos que afetaram a taxa de juros, com maior destaque para o final de 1989 e início de 1990, quando a caminhada para a hiperinflação foi interrompida pelo Plano Collor. 
Marcelo Fernandes \& Marcos de Bustamante Monteiro

Tabela 6. Resultados para o FGV-100

\section{Coeficientes Antes ICSS Depois ICSS}

\begin{tabular}{|c|c|c|}
\hline$\varphi$ & $0,0018(2,7287)$ & $0,0010(1.4788)$ \\
\hline$\phi_{1}$ & $0,1631(5,5802)$ & $0,2175(6.4955)$ \\
\hline$\phi_{2}$ & $0,1208(4,7864)$ & $0,0691(2.1827)$ \\
\hline$\omega \times 10^{3}$ & $0,0419(7,0226)$ & $0,2619(5.8915)$ \\
\hline$\alpha$ & $0,2485(14,8411)$ & $0,2047(7.8574)$ \\
\hline$\beta$ & $0.7515(79,4819)$ & $0,6981(21.1315)$ \\
\hline$\delta_{0} \times 10^{3}$ & $-\quad-$ & $-0,1458(-3.9697)$ \\
\hline$\delta_{1} \times 10^{3}$ & - & $-0,1817(-5.0114)$ \\
\hline$\delta_{2} \times 10^{3}$ & - & $-0,2268(-5.5289)$ \\
\hline Meia-Vida & $\infty$ & 7,78 \\
\hline$L(\theta)$ & $3.784,86$ & $3.903,58$ \\
\hline $\mathrm{BIC}$ & $-7.554,20$ & $-7.779,22$ \\
\hline$Q(16)$ & $19,854 \quad(0,2270)$ & $20,724 \quad(0,1890)$ \\
\hline$Q^{2}(16)$ & $7,4584(0,9156)$ & $5,7850(0,9716)$ \\
\hline
\end{tabular}

Apesar do modelo $\operatorname{ARIMA}(2,1,0)-\operatorname{IGARCH}(1,1)$ inicial ter passado em todos os testes de diagnóstico, o critério de informação bem inferior do segundo modelo confirma a melhoria expressiva acarretada pela introdução das dummies. Evidentemente, a persistência na volatilidade caiu violentamente no novo modelo, com uma meiavida próxima à encontrada para o índice da Bolsa de Valores de São Paulo. Por outro lado, a exemplo das outras séries estudadas, o comportamento da volatilidade é extremamente semelhante nos dois modelos, fato ilustrado nos gráficos 13 e 9 . 


\section{Conclusão.}

Analogamente aos efeitos de quebras estruturais nos testes de raíz unitária, instabilidades na variância nâo-condicional podem superestimar a persistência na volatilidade, induzindo a aceitação nãojustificada de modelos IGARCH. Com o intuito de fornecer um método consistente para a análise de persistência, recorreu-se aqui às técnicas de detecção de mudanças súbitas na variância, em particular, ao algoritmo ICSS desenvolvido por Inclán \& Tiao (1994). Ao contrário da metodologia de Lastrapes (1989), o procedimento proposto parte da informação contida na própria amostra para identificar de forma isenta os pontos de instabilidade no segundo momento.

Para a identificação das mudanças de regime na variância nãocondicional, deve-se inicialmente filtrar os dados originais via a estimação de um modelo de variância condicional heterocedástico. Em seguida, aplica-se o algoritmo ICSS para os resíduos padronizados, obtendo-se as subamostras correspondentes às variáveis binárias que devem ser introduzidas para captar os choques exógenos na variância não-condicional. Deste modo, consegue-se captar a heterocedasticidade condicional da série, destacando à presença de quebras estruturais e avaliando de maneira mais precisa a persistência dos choques na volatilidade.

A aplicação empírica realizada encontrou evidências de que os planos de estabilização no Brasil impactaram de modo significativo a volatilidade das séries financeiras. Neste contexto, cabe destacar os violentos impactos do Plano Collor I e II em todas as séries estudadas. Em geral, o impacto dos planos econômicos podem resultar em instabilidades na variância não-condicional das séries, de modo que as estimações preliminares apresentem evidências a favor de modelos IGARCH para descrever o comportamento da volatilidade. Após a incorporação de dummies para representá-los, a persistência na volatilidade é violentamente reduzida e não sendo necessário recorrer a modelos integrados na variância. A única exceção con- 
sistiu no processo inflacionário, que apresentou fortes evidências de integração na variância, apesar de aparentemente não ser integrado na média.

Submetido em Janeiro de 1996. Revisado em Julho de $199 \%$.

\section{Referências}

Bollerslev, T. 1986. "Generalized autoregressive conditional heteroskedasticity." Journal of Econometrics 31: 307-327.

Bollerslev, T. \& J. Wooldridge. 1992. "Quasi-maximum likelihood estimation and inference in dynamic models with time varying covariances." Econometric Reviews 11: 143-172.

Chou, R.Y. 1988. "Volatility persistence and Stock valuations: some empirical evidence using GARCH." Journal of Applied Econometrics 3: 279-294.

Diebold, F.X. 1986. "Modeling the persistence of conditional variances: a comment." Econometric Reviews 5: 51-56.

Ding, Z.; C.W.J. Granger \& R.F. Engle. 1993. "A long memory property of stock market returns and a new model." Journal of Empiracal Finance 1: 83-106.

Druck, S. 1991. "Um estudo de aspectos da incerteza do processo inflacionário brasileiro através de modelos de séries temporais com variância condicional." Dissertação de Doutorado Inédita. COPPE/UFRJ.

Engle, R.F. \& T. Bollerslev. 1986. "Modelling the persistence of conditional variances." Econometric Reviews 5: 1-50.

Fernandes, M. 1994. "Volatilidade na bolsa de valores do Rio de Janeiro." Anais do XVI Encontro Brasileiro de Econometria: 207-229. 
Flôres R.G., M.B. Monteiro \& A. Szafarz 1994. "Exchange rate volatility in high inflation economies: an econometric study of Poland and Brazil." Economics of Planning 27: 277-292.

Friedman, M. 1977. "Nobel lecture: inflation and unemployment." Journal of Political Economy 85: 451-472.

Geweke, J. \& S. Porter-Hudak. 1983. "The estimation and application of long memory time series models." Journal of Time Series Analysis 4: 221-238.

Hamilton, J.D. 1988. "Rational expectations econometrics analysis of changes in regime: an investigation of the term structure of interest rates." Journal of Economic Dynamics and Control 12: $385-424$.

Hamilton, J.D. \& R. Susmel 1992. "Autoregressive conditional heteroskedasticity and changes in regime." University of California at San Diego.

Hentschel, L. 1995. "All in the family: nesting symmetric and asymmetric GARCH Models." Journal of Financial Economics 39: 71-104.

Inclán, C. 1991 "Retrospective detection of sudden change of variance in time series." University of Chicago.

Ínclan, C. \& G.C. Tiao 1994. "Use of cumulative sums of squares for retrospective detection of changes of variance." Journal of the American Statistical Association 89: 913-923.

Issler, J.V. 1991. "Inflation level and uncertainty: evidence using Brazilian data." Revista de Econometria 45: 478-487.

Lastrapes, W.D. 1989. "Exchange rate volatility and U.S. monetary policy: an ARCH application." Journal of Money, Credit, and Banking 21: 66-77.

Lamoureux, C.G. \& W.D. Lastrapes 1992. "Persistence in variance, structural change and the GARCH model." Journal of Business and Economic Statistics 8: 225-234. 
Marcelo Fernandes \& Marcos de Bustamante Monteiro

Lumsdaine, R.L. 1996. "Consistency and asymptotic normality of the quasi-maximum likelihood estimator in $\operatorname{IGARCH}(1,1)$ and covariance stationary GARCH $(1,1)$ models." Econometrica 64: 575-596.

Nelson, D.B. 1991. "Conditional heteroskedasticity in asset returns: a new approach." Econometrica 59: 347-371.

Noh, J.; R.F. Engle \& A. Kane. 1994. "Forecasting volatility and option prices of the S\&P 500 index." Journal of Derivatives 7: $17-30$.

Phillips, P.C.B. \& P. Perron. 1988. "Testing for a unit root in time series regression." Biometrika 75: 335-346. 
Um Procedimento para Análise de Persistência na Volatilidade

Gráfico 1 - Variância Condicional Estimada para a Taxa de Câmbio

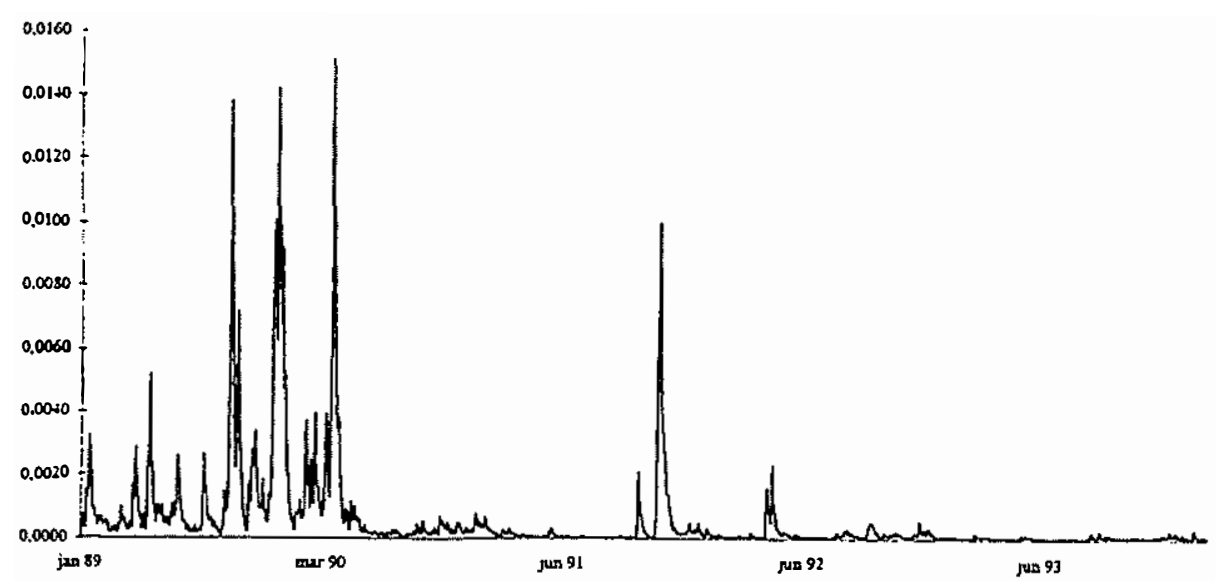

Gráfico 2 - Variância Estimada parar a Taxa de Câmbio após o Algoritmo ICSS

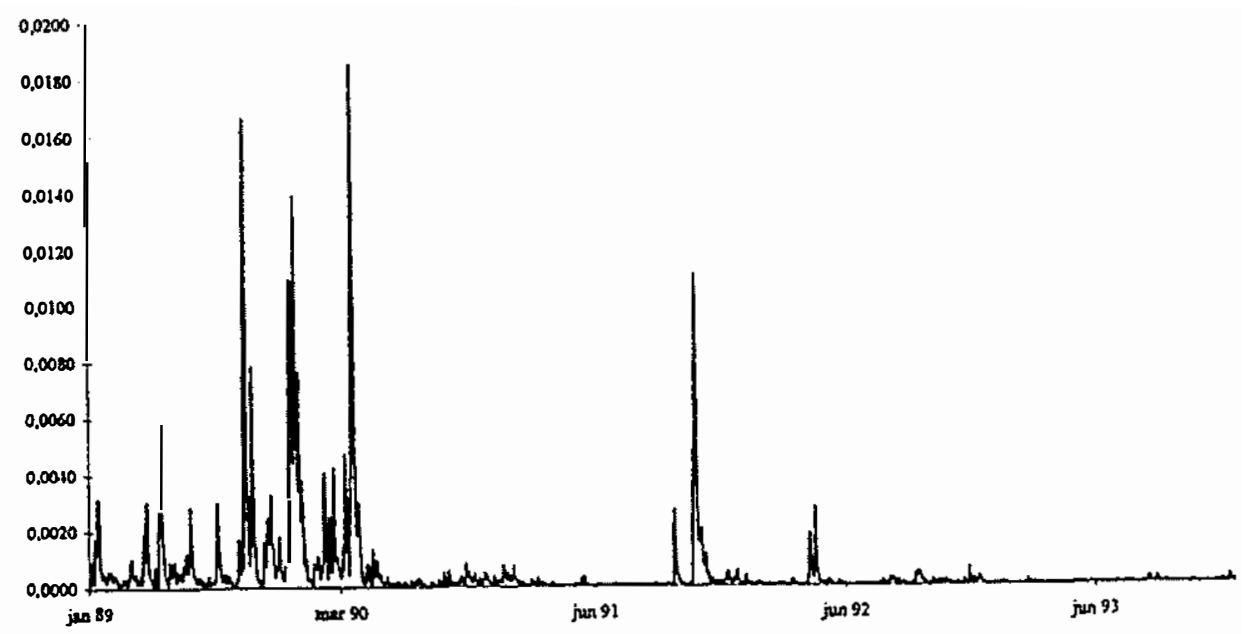


Marcelo Fernandes \& Marcos de Bustamante Monteiro

Gráfico 3a - Variância Condicional Estimada para a Taxa de Inflação

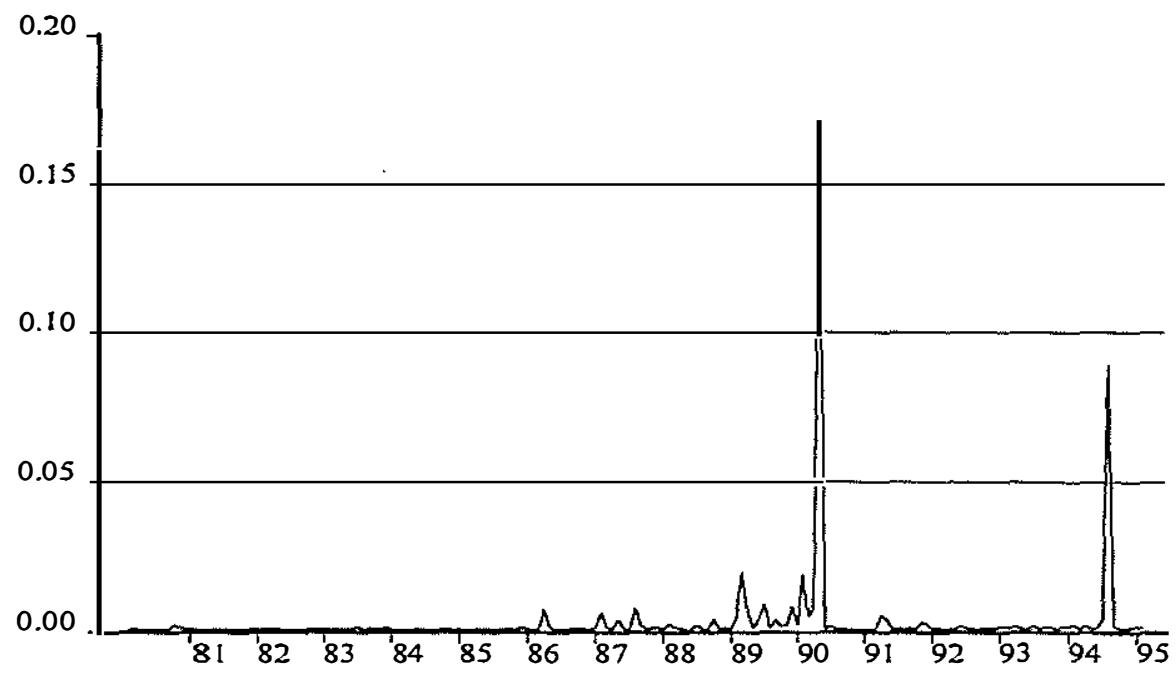

Gráfico 3b - Variância Condicional da Taxa de Inflação (86-95)

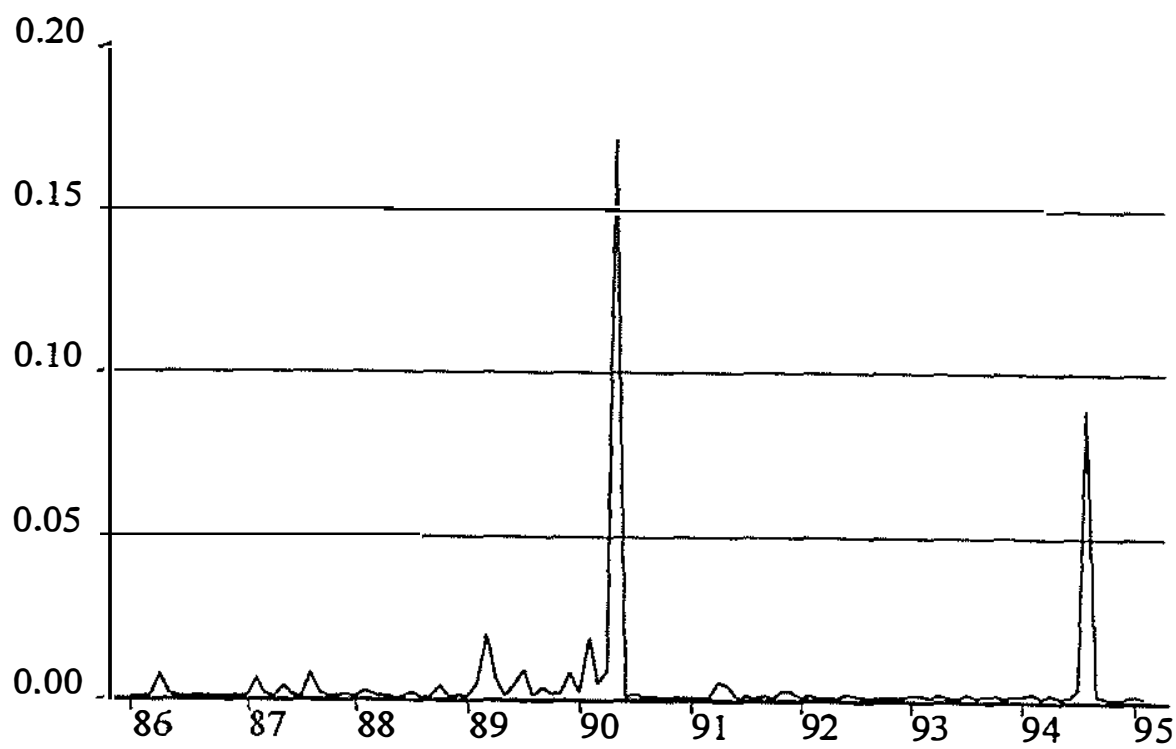

Revista de Econometria 17 (1) Maio 1997 
Um Procedimento para Análise de Persistência na Volatilidade

Gráfico 4a - Variância Cond. Estimada para Taxa de Inflação após Algoritmo ICSS

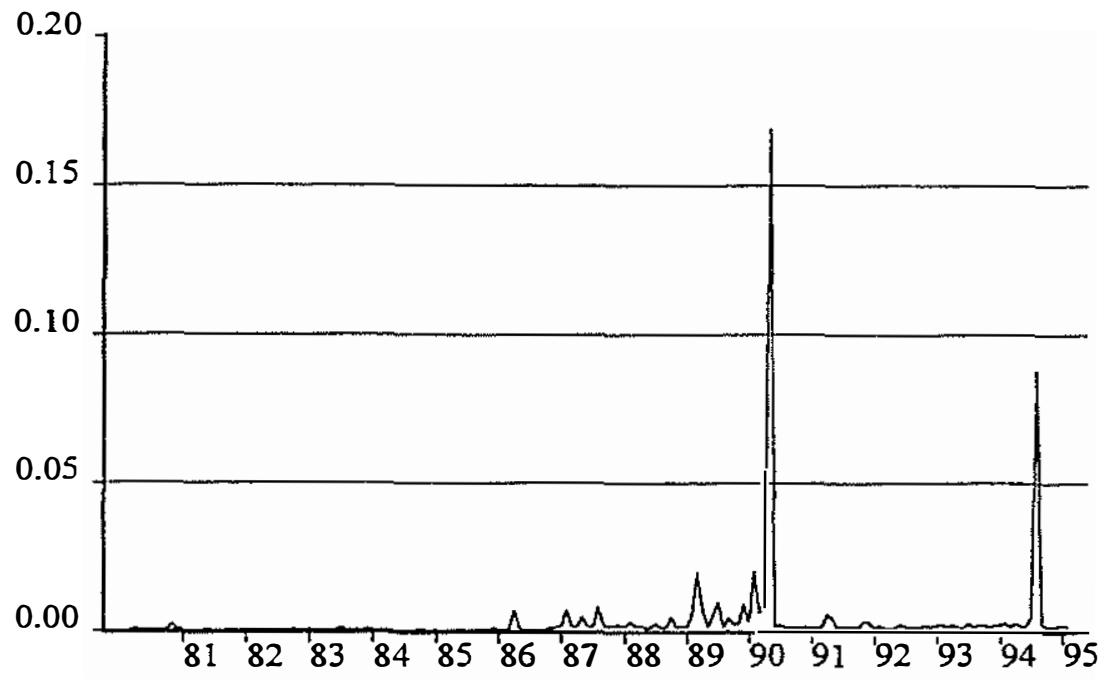

Gráfico 4b - Variância Cond. da Taxa de Inflação após Algoritmo ICSS (86-95)

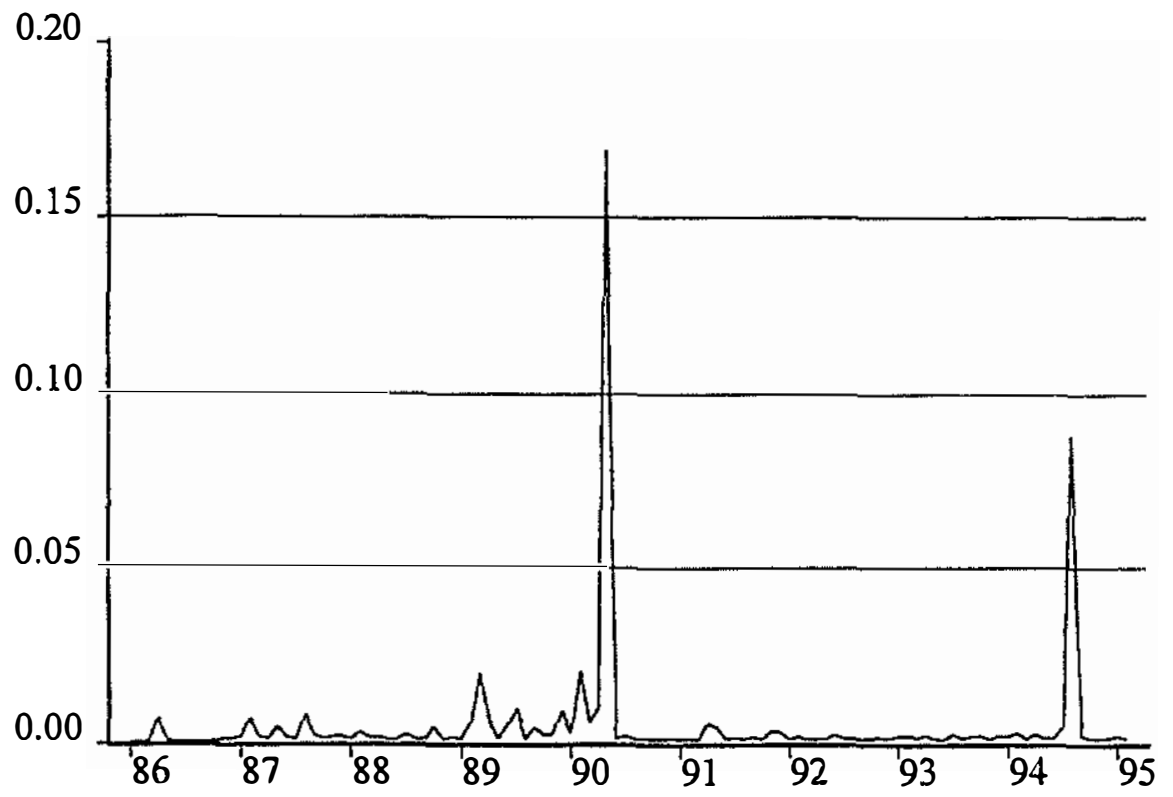


Marcelo Fernandes \& Marcos de Bustamante Monteiro

Gráfico 5 - Variância Condicional Estimada para a Taxa de Juros

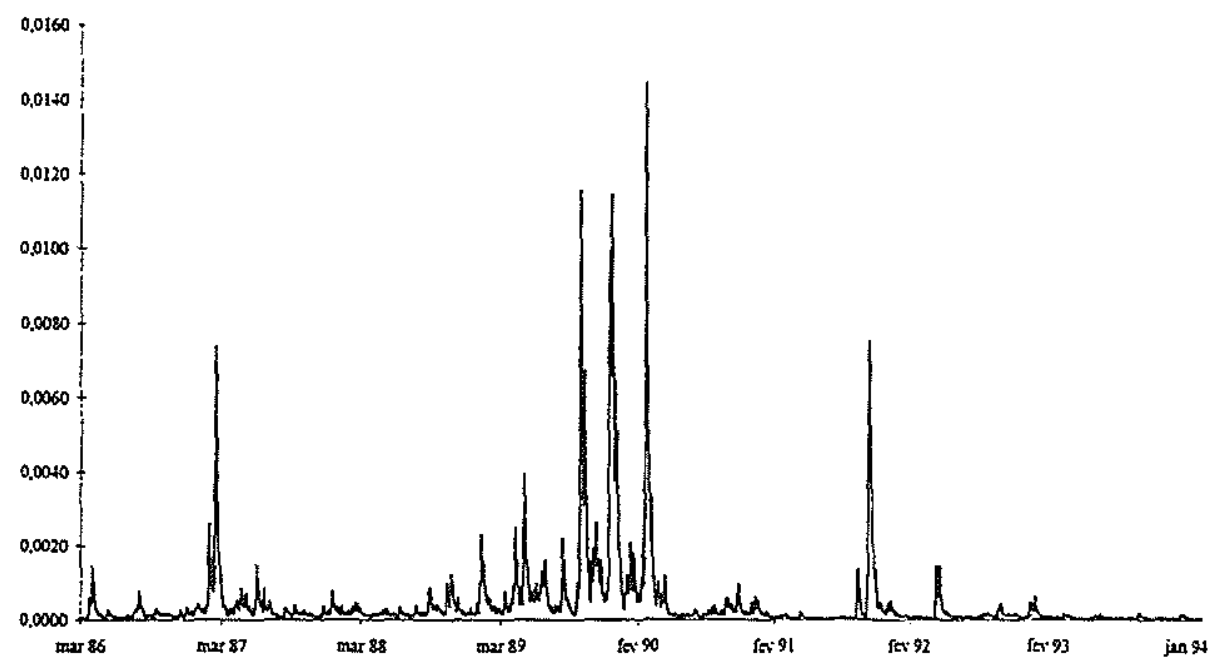

Gráfico 6 - Variância Cond. Estimada para Taxa de Juros após Algoritmo ICSS

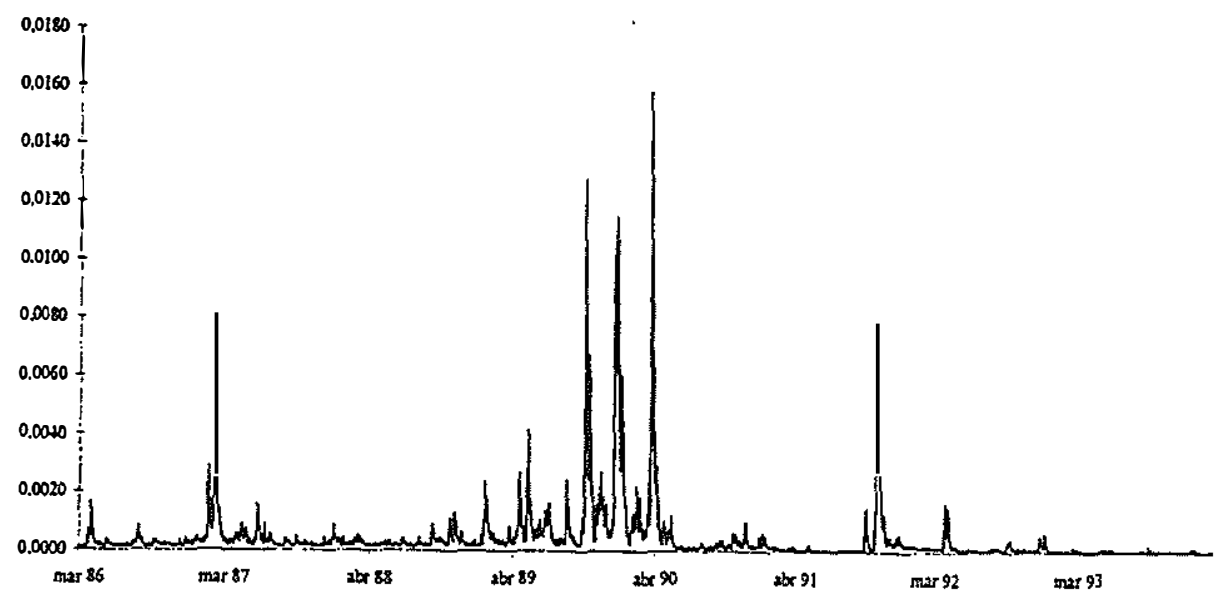

Revista de Econometria 17 (1) Maio 1997 
Um Procedimento para Análise de Persistência na Volatilidade

Gráfico 7 - Variância Condicional Estimada para o IBOVESPA

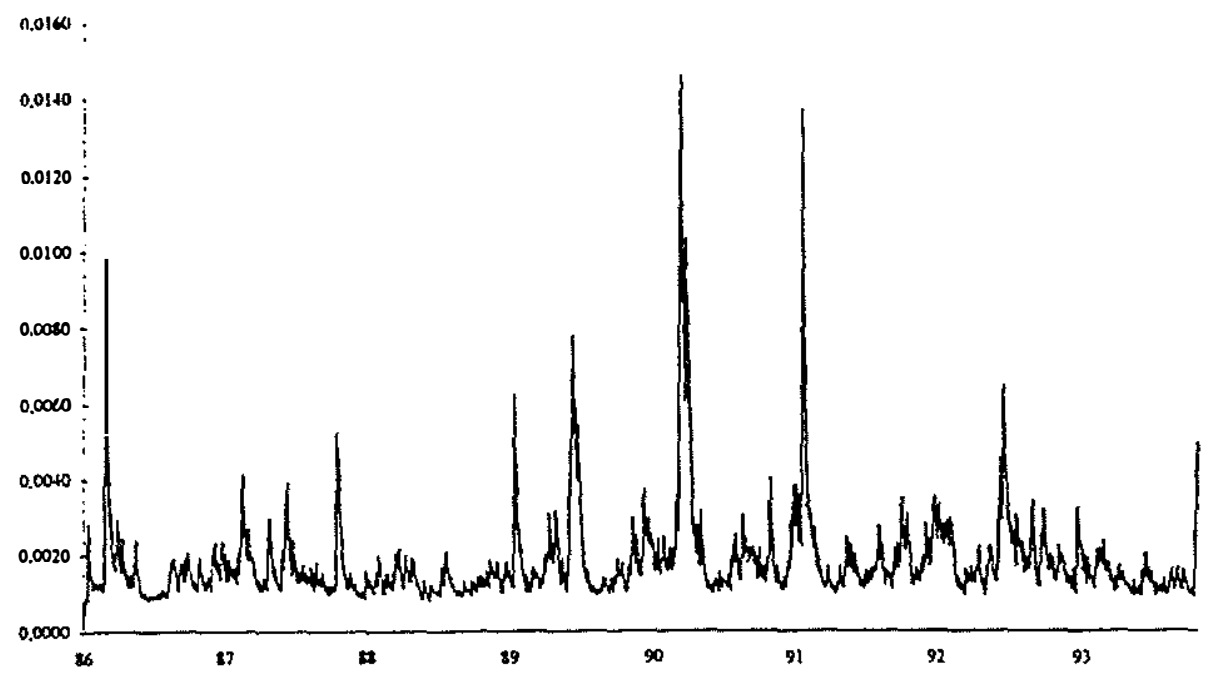

Gráfico 8 - Variância Condicional Estimada para o FGV-100

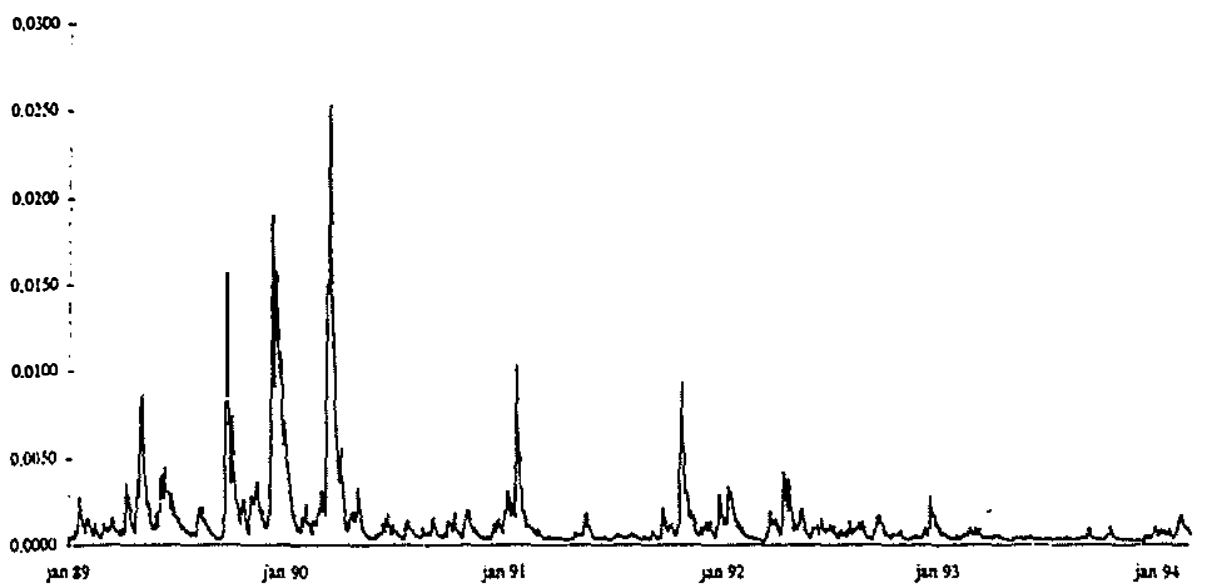


Marcelo Fernandes \& Marcos de Bustamante Monteiro

Gráfico 9 - Variância Cond. Estimada para o FGV-100 após Algoritmo ICSS

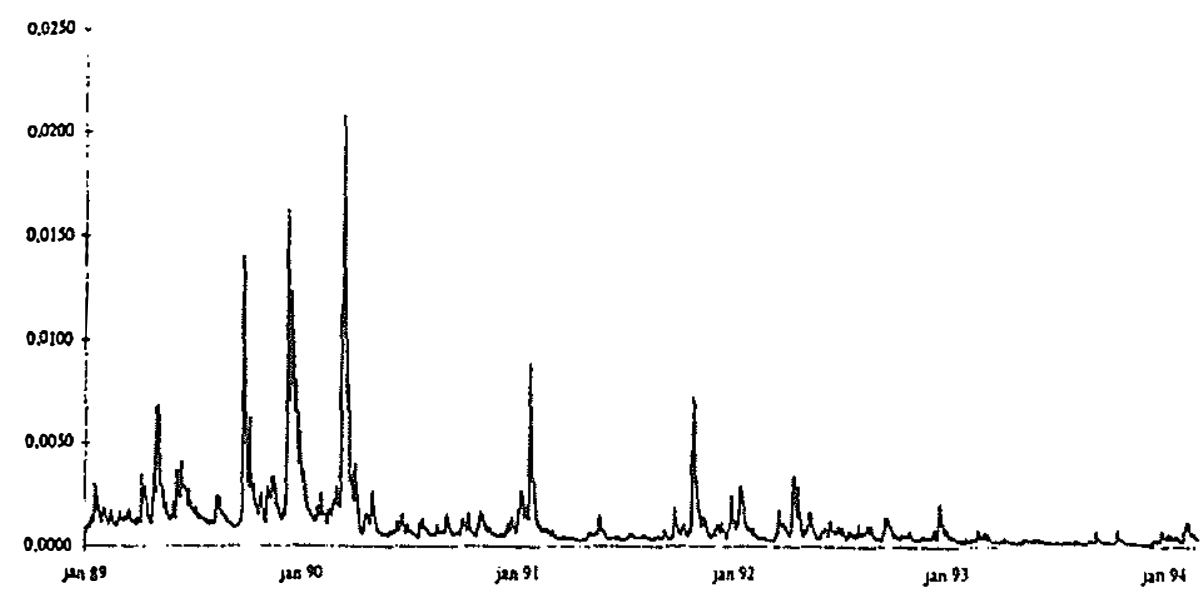


